\title{
Olive Mill Waste Water Added Alginate Films for Antibacterial Food Packaging Applications
}

\author{
Nil Erge Akkaya, \\ Istanbul Technical University, Faculty of Science and Literature, \\ Maslak, 34485 Istanbul, Turkey \\ Nurcan Ozcan, \\ Istanbul Technical University, Faculty of Science and Literature, \\ Maslak, 34485 Istanbul, Turkey \\ Ayse Saygun, (Corresponding author) \\ Istanbul Technical University, Faculty of Chemical and Metallurgical Engineering, \\ Maslak, 34485 Istanbul, Turkey \\ E-mail: gocmenay@itu.edu.tr \\ Nese Sahin Yesilcubuk, \\ Istanbul Technical University, Faculty of Chemical and Metallurgical Engineering, \\ Maslak, 34485 Istanbul, Turkey \\ Halit Turkmen \\ Istanbul Technical University, Faculty of Aeronautics and Astronautics, \\ Maslak, 34485 Istanbul, Turkey
}

\begin{abstract}
Alginate films are edible and biodegradable polysaccharides which are new food packaging candidates, however they do not have any antibacterial activity, which limits their applications. On the other hand, olive mill waste water (OMWW), which contains biophenols in its composition is a powerful antimicrobial material. The goal of the current study was to produce antibacterial alginate films making use of olive mill waste water for packaging applications and to investigate the physical and antibacterial properties of the prepared films.

In this work, olive mill waste water and glycerol added alginate films were prepared by the solvent cast method. Their antibacterial properties were investigated by the agar disc diffusion method, mechanical properties by tensile stretching and optical properties by UV-visible spectrometry.

Based on the results, pure alginate films and alginate-glycerol films didn't exhibit antibacterial activity whereas olive mill waste water addition resulted with antibacterial activity. In terms of mechanical properties, addition of olive mill waste water decreased the tensile strength by $50 \%$ and increased the elongation at break values of alginate films by more than $100 \%$ compared to pure alginate films and by $100 \%$ compared to alginate glycerol films, resulting in soft and flexible films.

Dark colored, UV barrier, antibacterial films are especially appropriate for UV protective biodegradable food packaging applications. This is the first application of olive mill waste water in a useful product.
\end{abstract}

Keywords Antibacterial food packaging, olive mill waste water, alginate films, biodegradable

DOI: $10.7176 / J S T R / 6-07-07$

\section{Introduction}

Food packaging is essential to protect food from contamination, to increase its shelf life, and to provide information about the product. Synthetic packaging materials are being widely used but waste of these materials cause environmental problems, so biodegradable packaging materials are started to be preferred (Cha and Chinnan 2004).

Polysaccharides are new candidates for replacing the synthetic packaging materials. Sodium alginate, which is all-purpose biodegradable polymer, is a linear polysaccharide, which due to its biocompatible and biodegradable nature is a good candidate for food packaging applications as a thin protective layer. It has also limited moisture, fine fats, oils and good oxygen barrier properties and it can provide 
protection against lipid oxidation and prevent shrinkage, and microbial contamination (Tavassoli-Kafrani et al. 2016).

In literature, antimicrobial properties of nisin, lysozyme, ethylene diamine tetra acetic acid, and grape fruit seed extract containing sodium alginate films were investigated by Cha and Chinnan (2004). Soy bean oil incorporated alginate films had improved microstructure homogeneity, transparency and reduced tensile strength and elongation at break and cinnamon bark oil improved antibacterial activity of sodium alginate films (Zhang et al. 2015). In the case of Oregano oil added alginate films the film's thickness and elongation at break increased, tensile strength and water vapor permeability decreased by $2.7-3.7 \%$ and films were found to be more effective on Gram positive bacteria than on Gram negative bacteria (Benavides et al. 2012).

Olive oil production has a great economical value in Mediterranean countries and $95 \%$ of world's olive oil is produced in these countries (Delisi et al. 2016). As a result of olive milling process, pomace and olive mill waste water (OMWW) are produced. OMWW is composed of $83-96 \%$ water, 3.5-15\% organics and $0.5-2 \%$ mineral salts. It has a low $\mathrm{pH}$ and high organic material content and high suspended solid content (Kapellakis et al. 2012). Organic part has 1.0-8.0\% sugars, $0.5-2.4 \%$ nitrate containing compounds, $0.5-1.5 \%$ organic acids, $0.02-1.0 \%$ oils, $1.0-1.5 \%$ phenols and pectin (Greco Jr. et al. 1999). OMWW composition depends on the climate, soil properties, olive type and olive tree (Yakhlef et al. 2018). It contains biophenols (0.1-18 g/L) which are powerful antimicrobials (Delisi et al. 2016). Components of OMWW with antimicrobial activity are hydroxytyrosol, oleuropein, 4-hydroxybenzoic acid, vanillic acid, and p-coumaric acid (Leouifoudi et al. 2015; Perez et al. 1992; Obied et al. 2007; Leouifoudi et al. 2015; Yakhlef et al. 2018). OMWW creates a great environmental problem when discharged into the soil or rivers due to high organic materials in its composition (Yakhlef et al. 2018; Obied et al. 2007). Despite the environmental problems, valorization of OMWW in food packaging materials might be possible due to its antimicrobial property.

In this work, OMWW added alginate films were prepared and the transparency, water vapor permeability, mechanical and antibacterial properties of the films were investigated. To the best of our knowledge, current study is the first research reporting the investigation of physical properties and antibacterial activity of OMWW in alginate films.

\section{Materials and methods}

\subsection{Materials}

Sodium alginate (low viscosity, Aldrich) and glycerol (Gly), (Merck, anhydrous, purity $\geq 99.5 \%$, boiling point $290^{\circ} \mathrm{C}$ ) were used as received. OMWW was obtained from a local producer in Ayvalık, Turkey. Gram-positive Staphylococcus aureus (S.aureus) ATCC 6538, Gram negative

Escherichia coli (E. coli) ATCC 43888 bacterial strains were cultured in nutrient broth and nutrient agar (Merck, Darmstadt, Germany) medium.

\subsection{Film preparation}

Films were prepared with distilled water. OMWW was centrifuged at $6000 \mathrm{rpm}$ for 60 minutes and filtered by a filter paper to remove the solid part then filtered through a $25 \mu \mathrm{m}$ microfilter. Film compositions were prepared according to Table 1 and $30 \mathrm{~mL}$ of solutions were poured into $8.5 \mathrm{~cm}$ diameter polystyrene petri dishes. Solutions were dried in an air ventilated oven at $60^{\circ} \mathrm{C}$ overnight. All experiments were performed at relative humidity level of $38 \pm 1 \%$ and at $25^{\circ} \mathrm{C}$.

Table 1. Film compositions of glycerol, OMWW added alginate films

\begin{tabular}{|c|c|c|c|c|}
\hline Film No. & Alginate $(\mathrm{g})$ & Water $(\mathrm{mL})$ & Glycerol $(\mathrm{g})$ & OMWW $(\mathrm{mL})$ \\
\hline $\mathbf{1}$ & 1 & 100 & - & - \\
\hline $\mathbf{2}$ & 1 & 100 & 0.3 & - \\
\hline $\mathbf{3}$ & 1 & 95 & - & 5 \\
\hline $\mathbf{4}$ & 1 & 95 & 0.3 & 5 \\
\hline
\end{tabular}

\subsection{Physical properties}

\subsubsection{Film thickness and transparency}

The thickness of each film was determined using a digital micrometer $(0-25 \mathrm{~mm}, 1 \mu \mathrm{m}$ sensitivity) at three randomly selected points of the film. The mean value of these three measurements was used to determine the physical and mechanical properties of the films. A Shimadzu double beam spectrophotometer UV-150-02 (Japan) was used for UV transmittance measurements. Film samples (1.5

74 I P a g e 
$\mathrm{cm} \times 4 \mathrm{~cm}$ ) were placed in the cells and measured against air. Film transmittance was calculated according to following equation (1) (Han and Floros 1997).

Transmittance $=10^{-\mathrm{Abs}}$

\subsubsection{Water vapor permeability}

Water vapor permeability (WVP) values of the films were calculated gravimetrically according to the ASTM, E96-95 standard (Zhang et al. 2015). A small cup $(20 \mathrm{~mL})$ with water and a magnetic follower was covered with a sample film $\left(1 \mathrm{~cm}^{2}\right)$ and the water in the cup was continuously stirred. The water level was $2 \mathrm{~cm}$ below the film. The air above the cups was ventilated to prevent the formation of a stagnant vapor layer above the films. Cups were periodically weighed after 1, 2, 3, 4, 5 hours and at the $24^{\text {th }}$ hour. The weight loss was plotted against time and the graph's linear slope was considered as water vapor transmission rate (WVTR). WVP was determined according to the following equation.

$\mathrm{WVP}=\frac{\mathrm{WVTR} \times \mathrm{L}}{\Delta \mathrm{P} \times \mathrm{A}}$

Here $\mathrm{A}$ is the film area $\left(\mathrm{m}^{2}\right), \mathrm{L}$ is the average film thickness $(\mathrm{mm})$, and $\Delta \mathrm{P}$ is the partial pressure difference through the film $(\mathrm{Pa})$.

\subsection{Mechanical properties}

Tensile strength and \% elongation of the films were determined at room temperature by using MTS Universal Testing Machine (322, the model number) according to ASTM standard method D882-18. Films were cut into rectangular shape $(66 \mathrm{~mm}$ x $12 \mathrm{~mm}$ x $50 \mu \mathrm{m})$ and mounted between the corrugated tensile grips of the instrument. To avoid the deformation caused by the grip structure, the two ends of the films, which come in contact with the grips, were covered with plastic films using epoxy resin and left to dry for 12 hours. Initial grip spacing and cross head speed were $30 \mathrm{~mm}$ and $2 \mathrm{~mm} / \mathrm{min}$, respectively. Force and elongation values were monitored until break. The tensile strength was expressed as the force divided by the initial cross-sectional area of the sample (Eq. 3) and \% elongation at break values were obtained by dividing the elongation at break values to the original length (Eq. 4 ). 4 to 6 samples were tested from each composition in order to get statistically significant results.

$$
\begin{aligned}
& \mathrm{TS}=\frac{\text { Maximum force at break }(\mathrm{N})}{\text { Initial cross sectional area of film }\left(\mathrm{mm}^{2}\right)} \\
& \% \mathrm{E}=\frac{\text { Elongation at break }(\mathrm{mm})}{\text { Initial length }(\mathrm{mm})} \times 100
\end{aligned}
$$

\subsection{Antibacterial activity}

The antibacterial activity of films were measured using the agar disc diffusion method. The antibacterial activities of films were investigated for inhibitory effects towards Gram positive bacteria, S. aureus and Gram negative bacteria, E. coli. Overnight cultures were prepared by picking a single colony from a stock culture plate, inoculating into sterile nutrient broth (Merck, Darmstadt, Germany) and incubating at $37^{\circ} \mathrm{C}$ for $16 \mathrm{~h}$. An aliquot of $0.1 \mathrm{~mL}$ overnight culture was evenly spread on nutrient agar plate surface with drigalski. For testing the antibacterial activity of the film samples, two pieces of circular films which were aseptically cut into circles having diameter of $0.6 \mathrm{~cm}$ were placed in each petri dish. The plates were incubated at $37^{\circ} \mathrm{C}$ for $48 \mathrm{~h}$ and the area of microbial inhibition zone was measured (Tepe et al. 2005; Obied et al. 2007; Balti et al. 2017).

\section{Results and Discussion}

\subsection{Film thickness and transmittance}

Thickness of the films together with mechanical and physical properties are given in Table 2. As the amount of additives were increased film thickness also increased.

Figure 1 . is a photo of films $(2-4)$ showing their transparency. $1^{\text {st }}$ and $2^{\text {nd }}$ films which did not contain OMWW were not visually different so only $2^{\text {nd }}$ film is shown in the figure. The OMWW containing $3^{\text {rd }}$ and $4^{\text {th }}$ films had brownish hues. Third film with OMWW and without glycerol was the darkest one. The spectroscopic results in Figure 2 quantify these results. The pure alginate film (Number 1) has the highest and alginate OMWW film (Number 3) one has the lowest transparency at all wavelengths. Both OMWW films were opaque in the UV $(200-350 \mathrm{~nm})$ band. This is a useful property in food packaging especially 
where UV exposure accelerates ripening or spoilage of various fruits and vegetables including olive products (Caponio et al. 2005).

Table 2. Physical properties of glycerol, OMWW added alginate films

\begin{tabular}{|c|c|c|c|c|}
\hline Film No. & $\mathbf{1}$ & $\mathbf{2}$ & $\mathbf{3}$ & $\mathbf{4}$ \\
\hline Alginate $(\mathrm{g})$ & 1 & 1 & 1 & 1 \\
\hline Water $(\mathrm{mL})$ & 100 & 100 & 95 & 95 \\
\hline Glycerol $(\mathrm{g})$ & - & 0.3 & - & 0.3 \\
\hline OMWW $(\mathrm{mL})$ & - & - & 5 & 5 \\
\hline Thickness (mm) & $0.048 \pm 0.001$ & $0.064 \pm 0.001$ & $0.068 \pm 0.001$ & $0.085 \pm 0.001$ \\
\hline Tensile Strength (MPa) & $71.2 \pm 10.2$ & $29.09 \pm 3.5$ & Brittle & $14.53 \pm 2.97$ \\
\hline E, \% & $3.5 \pm 0.8$ & $4.50 \pm 2.7$ & Brittle & $9.56 \pm 2.7$ \\
\hline WVTR (g/s) & $1.53 \times 10^{-6}$ & $5.51 \times 10^{-6}$ & $5.87 \times 10^{-6}$ & $7.16 \times 10^{-6}$ \\
\hline WVP $(\mathrm{g} / \mathrm{s} . \mathrm{m} . \mathrm{Pa})$ & $0.5 \times 10^{-9}$ & $1.80 \times 10^{-9}$ & $2.03 \times 10^{-9}$ & $2.33 \times 10^{-9}$ \\
\hline
\end{tabular}

\subsection{Water vapour permeability}

The water vapour transfer and water vapour permeability values of the films as seen in Table 2, first film is obtained from pure alginate. The $2^{\text {nd }}$ film is glycerol added alginate film. The $3^{\text {rd }}$ film contains OMWW, $4^{\text {th }}$ film contains glycerol and OMWW. The WVTR and WVP values increased from second to forth. Additives increased the WVTR and WVP of the films. For packaging applications alginate based films have WVP values in the range of $0.410^{-10}-1.410^{-9} \mathrm{~g} / \mathrm{s} . \mathrm{m} . P a$ (Rhim 2004; Wang et al. 2017; Yang et al. 2016; Olivas and Barbosa-Canovas 2008; Liling et al. 2016), our films have slightly higher WVP values, still suitable for packaging applications.

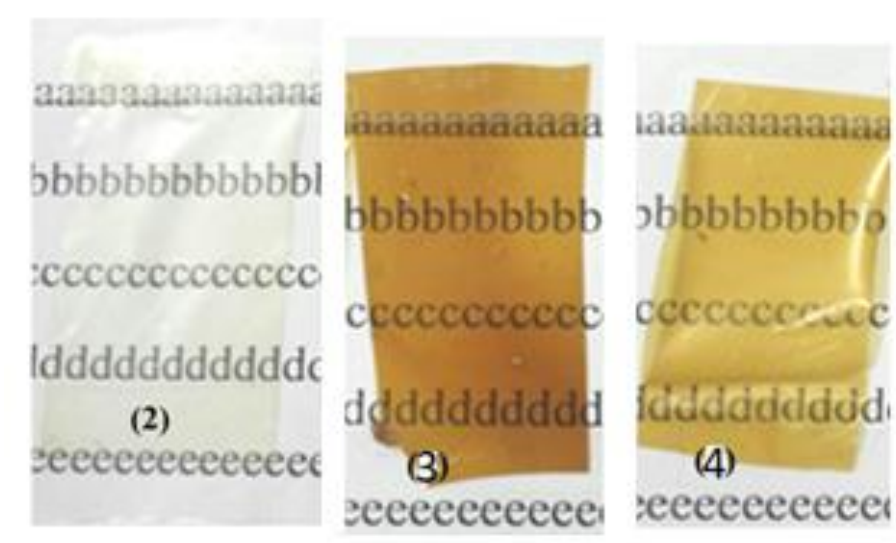

Figure 1. Color and transparency of alginate films. Film incorporated with glycerol (No: 2), with OMWW (No: 3), with glycerol and OMWW (No: 4). (The letters are arbitrary and are used to demonstrate the visual transparency of the films.) 


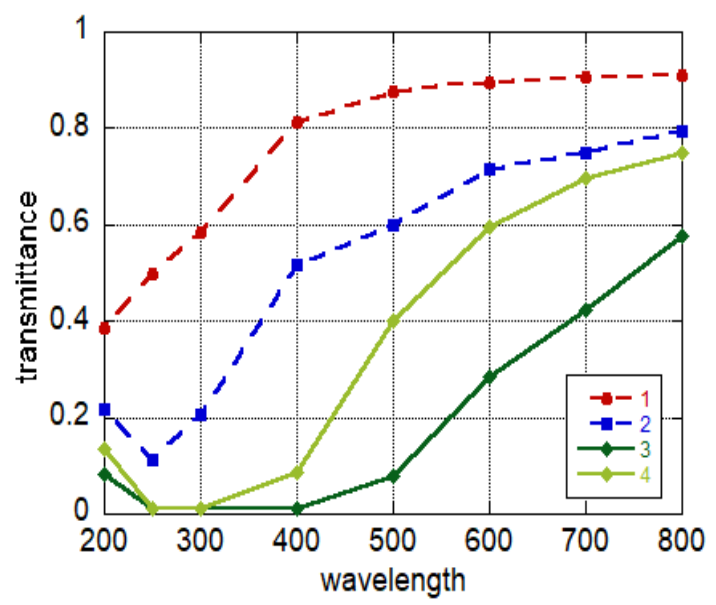

Figure 2. Transmittance of, pure alginate film (No: 1), glycerol added alginate film (No: 2), OMWW added alginate film (No: 3) glycerol and OMWW added alginate film (No: 4).

\subsection{Mechanical properties}

Stress-strain graphics of $1^{\text {st }}, 2^{\text {nd }}$ and $4^{\text {th }}$ films are shown in Fig. 3. The $3^{\text {rd }}$ film was extremely brittle and fragmented without any measurable stretching. Calculated tensile strength and \% elongation at break values are given in Table 2. Pure alginate film has a high tensile stress but low \% elongation at break. Addition of glycerol (No: 2), glycerol and OMWW (No: 4) decreased the tensile strength and increased the $\%$ elongation at break values. In the glycerol added films, replacing $5 \%$ of the water in the film forming solution by OMWW decreased the tensile strength and increased the elongation at break value considerably, resulting in soft and flexible films.

In Figure 3, pure alginate film is strong and brittle (1), glycerol addition produced softer films (2). Alginate-OMWW film become very weak and brittle to be tested and we cannot add number 3 to the figure and $4^{\text {th }}$ one is alginate-glycerol-OMWW sample and it is soft. These are expected results. StressStrain values of alginate and alginate-glycerol films are in line with the value quoted in literature (Su Giz, et al., 2020; Su Giz et al., 2019). OMWW addition is expected to weaken and soften the films.

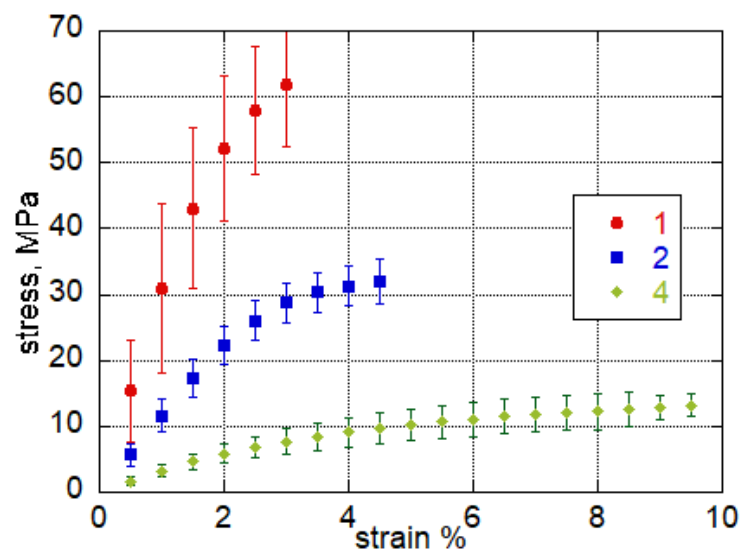

Figure 3: Stress-strain plots of pure alginate (1), Gly added alginate (2), Gly and OMWW added alginate (4) films. Third film was too brittle and is not displayed here. 
Table 3. Area of the inhibitory zones of pure additives and film samples

\begin{tabular}{|c|c|c|}
\hline \multirow{2}{*}{ Fim additive } & \multicolumn{2}{|c|}{ Area of inhibitory zone $\left(\mathbf{c m}^{2}\right)$} \\
\cline { 2 - 3 } & $\boldsymbol{E}$. coli & S. aureus \\
\hline OMWW $(100 \mu \mathrm{L})$ & $0.65 \pm 0.0011$ & $1.04 \pm 0.0223$ \\
\hline Glycerol $(100 \mu \mathrm{L})$ & $0.35 \pm 0.0011$ & $0.22 \pm 0.0001$ \\
\hline Sample number & & \\
\hline 1 & - & - \\
\hline 2 & - & - \\
\hline 3 & $0.10 \pm 0.0007$ & $0.85 \pm 0.0023$ \\
\hline 4 & $0.10 \pm 0.0001$ & $0.87 \pm 0.0009$ \\
\hline
\end{tabular}

\subsection{Antibacterial Activity Test}

Antibacterial activities of the additives, and the films were tested against Gram positive and Gram negative bacteria using the agar diffusion method. The sizes of the areas of inhibitory zones of samples are shown in Table 3. As can be seen from the table, glycerol which was used to increase film softness and flexibility also showed some inhibitory activity. In the Table $3,1^{\text {st }}$ film, pure alginate film and $2^{\text {nd }}$ film , glycerol added alginate film did not have any antibacterial activity. In the $3^{\text {rd }}$ OMWW film had inhibitory activity against $E$. coli and $S$. aureus. The antimicrobial activity of the glycerol and OMWW added $4^{\text {th }}$ film was very close to the $3^{\text {rd }}$ film .

\section{Conclusion}

OMWW addition to alginate films created dark colored films with low UV transmittance for protecting the foods from the adverse effects of UV radiation. Moreover, films had slightly higher VWP values, still suitable for packaging applications.

Pure alginate and glycerol added alginate films ( $1^{\text {st }}$ and $2^{\text {nd }}$ films) had no antibacterial activity. OMWW added films ( $3^{\text {rd }}$ and $4^{\text {th }}$ films) had activity against both E. coli and S.aureus. In terms of mechanical properties, the $3^{\text {rd }}$ film was brittle and was not suitable for packaging purposes. The OMWW and glycerol added $4^{\text {th }}$ film was not brittle, had antibacterial activity and appropriate properties for food packaging applications. We believe that valorization of OMWW in edible films would be beneficial for the environment as well as it will be used as a value added product.

\section{References}

Balti R., Mansour M.B., Sayari N., Yacoubi L., Rabaoui L., Brodu N., Massé A. (2017), Development and characterization of bioactive edible films from spider crab (Maja crispata) chitosan incorporated with Spirulina extract. Int. J. of Biological Macromolecules 105, 1464-1472.

Benavides S., Villalobos-Carvajal R., Reyes J.E. (2012), Physical, mechanical and antibacterial properties of alginate film: Effect of the crosslinking degree and oregano essential oil concentration. J Food Eng 110, 232-239. doi: 10.1016/j.jfoodeng.2011.05.023.

Caponio F., Bilancia M.T, Pasqualone A., Sikorska E., Gomes T. (2005), Influence of the exposure to light on extra virgin olive oil quality during storage. Eur Food Res Technol 221, 92-98. doi: 10.1007/s00217-004-1126-8.

Cha D.S, Chinnan M.S. (2004), Biopolymer-based antimicrobial packaging: A review. Crit Rev Food Sci Nutr 44, 223-237. doi: 10.1080/10408690490464276.

Delisi R, Saiano F, Pagliaro M, Ciriminna R. (2016), Quick assessment of the economic value of olive mill waste water. Chem Cent J 10, 8-12. doi: 10.1186/s13065-016-0207-7.

Greco, Jr G., Toscanoa, G., Cioffi, M., Gianfreda, L., Sannino, F. (1999), Dephenolisation of olive mill waste-waters by olive husk. Water Res 33, 3046-3050. doi: 10.1016/S0043-1354(99)000081. 
Kapellakis, I.E., Paranychianakis, N.V., Tsagarakis, KP, Angelakis, A.N. (2012), Treatment of olive mill wastewater with constructed wetlands. Water (Switzerland) 4, 260-271. doi: $10.3390 / \mathrm{w} 4010260$.

Leouifoudi, I., Harnafi, H., Zyad, A. (2015), Olive Mill Waste Extracts: Polyphenols Content, Antioxidant, and Antimicrobial Activities. Adv Pharmacol Sci 2015. doi: 10.1155/2015/714138.

Liling, G., Di Z., Jiachao, X., Xin, G., Xiaoting, F., Qing, Z. (2016), Effects of ionic crosslinking on physical and mechanical properties of alginate mulching films. Carbohydr Polym 136, 259-265. doi: 10.1016/j.carbpol. 2015.09.034.

Obied, H.K., Bedgood, Jr D.R., Prenzler, P.D., Robards, K. (2007), Bioscreening of Australian olive mill waste extracts: Biophenol content, antioxidant, antimicrobial and molluscicidal activities. Food Chem Toxicol 45, 1238-1248. doi: 10.1016/j.fct.2007.01.004.

Olivas, G.I., Barbosa-Cánovas, G.V. (2008), Alginate-calcium films: Water vapor permeability and mechanical properties as affected by plasticizer and relative humidity. LWT - Food Sci Technol 41, 359-366. doi:10.1016/j.lwt.2007.02.015.

Perez, J., De La Rubia, T., Moreno, J., Martinez, J. (1992), Activity of Olive Oil Waste Waters. Environ Toxicol Chem 11, 489-495.

Rhim, J.W. (2004), Physical and mechanical properties of water resistant sodium alginate films. $L W T$ - Food Sci Technol 37, 323-330. doi:10.1016/j.lwt.2003.09.008.

Su Giz, A., Berberoglu, M., Bener, S., Aydelik-Ayazoglu, S., Bayraktar, H., Alaca, B.E., Çatalgil-Giz, H. (2020), A detailed investigation of the effect of calcium crosslinking and glycerol plasticizing on the physical properties of alginate films. International Journal of Biological Macromolecules, 148, 49-55, doi: 10.1016/j.ijbiomac.2020.01.103.

Su Giz, A., Aydelik-Ayazoglu, S., Çatalgil-Giz, H. Bayraktar, H., Alaca, B.E. (2019), Stress relaxation and humidity dependence in sodium alginate-glycerol films. Journal of the Mechanical Behavior of Biomedical Materials, 100:103374, doi: 10.1016/j.jmbbm.2019.103374.

Tavassoli-Kafrani, E., Shekarchizadeh, H., Masoudpour-Behabadi, M. (2016), Development of edible films and coatings from alginates and carrageenans. Carbohydr Polym 137, 360-374.

Tepe, B., Daferera, D., Sokmen, A., Sokmen, M., Polissiou, M. (2005), Antimicrobial and antioxidant activities of the essential oils and various extracts of Salvia tomentosa Miller (Lamiaceae). Food Chem 90, 333-340.

Wang, L.F., Shankar, S., Rhim, J.W. (2017), Properties of alginate -based films reinforced with cellulose fibers and cellulose nanowhiskers isolated from mulberry pulp. Food hydrocolloids 63, 201-208.

Yakhlef, W., Arhab, R., Romero, C., Manuel, B.B., Antoninode, C.G-M., Eduardo, M.P. (2018), Phenolic composition and antimicrobial activity of Algerian olive products and by-products. $L W T$ - Food Sci Technol 93, 323-328. doi: 10.1016/j.lwt.2018.03.044.

Yang, M., Xia, Y., Wang, Y., Zhao, X., Xue, Z., Quan, F., Geng, C, Zhao, Z. (2016), Preparation and property investigation of crosslinked alginate/silicon dioxide nanocomposite films. $J$ Appl Polym Sci 133, 1-9. doi:10.1002/app.43489.

Zhang, Y., Ma, Q., Critzer, F., Davidson, M.P., Zhong, Q. (2015), Physical and antibacterial properties of alginate films containing cinnamon bark oil and soybean oil. LWT - Food Sci Technol 64, 423430. doi: 10.1016/j.lwt.2015.05.008. 